\title{
Oligometastatic breast cancer: where are we now and where are we headed? - a narrative review
}

\author{
Collin L. Kent ${ }^{1}$, Susan G. R. McDuff ${ }^{1}$, Joseph K. Salama ${ }^{1,2}$ \\ ${ }^{1}$ Department of Radiation Oncology, Duke University, Durham, NC, USA; ${ }^{2}$ Radiation Oncology Service, Durham VA Medical Center, Durham, NC, USA \\ Contributions: (I) Conception and design: JK Salama; (II) Administrative support: None; (III) Provision of study materials or patients: None; (IV) \\ Collection and assembly of data: All authors; (V) Data analysis and interpretation: All authors; (VI) Manuscript writing: All authors; (VII) Final \\ approval of manuscript: All authors. \\ Correspondence to: Joseph K. Salama, MD. Box 3085, Duke University Medical Center, Durham, NC 27710, USA. Email: joseph.salama@duke.edu.
}

\begin{abstract}
Metastatic breast cancer has traditionally been considered incurable, with treatments focused on systemic therapies and palliative local treatment. However, evidence is emerging that in some patients with limited metastatic disease, or "oligometastatic disease," often defined as five or fewer metastases diagnosed on imaging, aggressive metastasis-directed therapy (MDT) with surgery and/or hypofractionated imageguided radiation therapy (HIGRT) improves outcomes and may even be curative. This practice is becoming more common as evidence has grown to support the approach and as technology has made it more feasible. Treatment of certain oligometastatic breast cancers in particular (i.e., hormone receptor positive and boneonly metastases) may be especially useful given the long natural history of the disease in some of these patients. Recently, high quality data supporting ablative MDT in patients with oligometastatic disease has emerged from randomized trials for specific sites such as non-small cell lung cancer and prostate cancer, as well as from histology agnostic studies (i.e., SABR-COMET). However, randomized data in breast cancer specifically is currently lacking. Retrospective series and subgroup analysis from prospective trials have demonstrated improved outcomes with MDT for oligometastatic breast cancer. The ongoing phase II/III NRG BR002 trial seeks to provide the first randomized data to determine whether MDT in oligometastatic breast cancer improves outcomes. This may be especially important as improved systemic therapies such as targeted agents and immunotherapy prolong the disease course. Alternatively, if improved systemic therapies render patients disease free, MDT may not be necessary and only adds toxicity. However, MDT may also provide non-curative benefits for patients such as palliation of symptoms and extended time off systemic therapy. For now, aggressive MDT for certain favorable subgroups of oligometastatic breast cancer such as those with few metastases, hormone positive disease, and/or bone-only metastases is reasonable and may improve outcomes. We eagerly anticipate the results of NRG BR002 to further clarify the role of ablative therapy to all sites of disease in these patients.
\end{abstract}

Keywords: Radiofrequency ablation (RFA); liver resection; stereotactic ablative radiotherapy (SABR); transarterial chemotherapy (TACE); stereotactic body radiation therapy (SBRT); hypofractionated image-guided radiation therapy (HIGRT); oligometastases

Submitted May 17, 2020. Accepted for publication Jul 27, 2020.

doi: 10.21037/apm-20-1128

View this article at: http://dx.doi.org/10.21037/apm-20-1128

\section{Introduction}

Metastatic cancer has long been considered incurable, with treatment historically focused on systemic therapy to extend life and local therapy for palliation of symptoms (1). However, there has been growing interest in more aggressive metastasis-directed therapies as a clinically significant portion of patients $(\sim 25 \%)$ with limited metastases treated with curative intent have long disease- 
free survival (2-4). A recent international survey of more than 1,000 radiation oncologists found that more than $60 \%$ of respondents use ablative radiation therapy to treat patients with a limited number of metastases (5), with a similar use of surgical metastasectomy (6).

Despite the increasing adoption of aggressive metastasisdirected therapy (MDT), the topic warrants further discussion in a new era of recently published high quality studies both histology agnostic such as the recently updated SABR-COMET trial $(7,8)$, and disease specific trials in colorectal cancer (9), non-small cell lung cancer (NSCLC) $(10-12)$, prostate cancer $(13,14)$, and the ongoing phase II/III study, NRG BR002, randomizing patients with locally controlled four or fewer breast cancer metastases to standard systemic therapy with or without ablative metastasis-directed radiotherapy or surgery (15).

Additionally, in the context of new systemic therapies, in particular targeted therapies (16-20) and immunotherapy $(21,22)$, with the ability to destroy micrometastases and extend survival, metastasis-directed therapies for patients with limited metastatic disease may become more relevant. Alternatively, should the systemic therapies develop to a point where they render patients disease free, the addition of MDT would be moot, and only add to the risk of side effects. In this review, we will discuss the current understanding of oligometastatic disease, treatment of oligometastases, recent high-quality evidence supporting MDT in general and for breast cancer specifically, and considerations in the context of new systemic therapies. We present the following article in accordance with the NARRATIVE REVIEW reporting checklist (available at http://dx.doi.org/10.21037/apm-20-1128).

\section{What is the oligometastatic state?}

The oligometastatic state was first described by Hellman and Weichselbaum based on the spectrum theory of cancer spread (23). They postulated that metastatic disease occurs on a spectrum, including a clinically distinct, intermediate stage between locoregionally confined disease and widespread distant metastases. In this intermediate state, the disease may have a more indolent biology compared to later in the metastatic cascade (24). Given this, they hypothesized that treatment of all known cancer, both primary tumors and metastases, could lead to long disease-free intervals and potentially even cure.

The term oligometastasis means "few"-from the Greek "oligos"-metastases, however, an exact number of lesions is rarely defined. Most studies have limited inclusion to patients with five or fewer metastases (3), although some have included patients with up to eight (25). These criteria are based on imaging and may evolve with continued adoption of more sensitive imaging techniques such as PET/CT. The European Organization for Research and Treatment of Cancer (EORTC) and European Society for Radiotherapy and Oncology (ESTRO) have published recommendations for imaging-based diagnosis of oligometastatic disease (26-28), and together with the American Society for Radiation Oncology (ASTRO), ASTRO-ESTRO is currently working to establish a consensus definition.

While there is not yet a consensus definition for oligometastases, additional terms have been coined to further describe temporal patterns of cancer spread. The terms "de novo oligometastases," or "synchronous oligometastases," describe limited metastatic disease at the time of the initial cancer diagnosis and includes an untreated primary tumor in addition to limited metastases. The phrase "induced oligometastases" describes patients with widely metastatic disease in which most, but not all, metastases respond to systemic therapy, with a subsequent few remaining metastases (24). "Oligorecurrence" describes patients who develop limited metastases after initial definitive treatment (29). Finally, "oligoprogression" describes patients receiving systemic therapy who initially demonstrate response of all metastases to systemic therapy and subsequently experience progression in a limited number of disease sites while the rest remain controlled. It has been hypothesized that oligoprogression occurs when a limited number of tumor clonogens develop mutations that result in resistance to systemic therapy while the majority of metastases remain susceptible and controlled $(30,31)$.

\section{Are oligometastases common in breast cancer?}

While evidence supporting the oligometastatic state was sparse when Hellman and Weichselbaum first proposed their theory, since then numerous studies have demonstrated oligometastases to be fairly common. Among patients with metastatic breast cancer enrolled on major phase II and phase III clinical trials of systemic therapies, approximately $50 \%$ present with two or fewer clinically detected metastases (32-37). Similar rates of oligometastases are seen in patients with NSCLC, melanoma, prostate cancer, and colorectal cancer (38-41).

Additionally, patients with oligometastatic breast cancer have been shown to have better outcomes than patients with widespread metastases. Data from the 1980s 
demonstrated that among patients with metastatic breast cancer, the presence of five or fewer metastases was an independent predictor of survival (42). More recent data has shown that patients with early-stage breast cancer who progressed with five or fewer metastases have improved five-year $(59.6 \%$ vs. $11.6 \%)$ and median survival (107.7 vs. 22 months; $\mathrm{P}=0.001)$ compared to patients with more than five metastases (43). Similarly, studies have shown improved outcomes for patients with oligometastatic prostate cancer and NSCLC compared to patients with widespread disease $(38,39,44)$. Taken together, it is clear that oligometastatic disease, including breast cancer, is relatively common and has meaningful clinical significance.

\section{How are oligometastases treated?}

\section{Surgery}

MDT for oligometastases was first described in surgical literature. Large series have examined outcomes after resection of lung (45), liver (46), adrenal (47), and brain (48) metastases and demonstrated better-than-expected longterm disease control and survival for select patients. One retrospective series of 467 patients with metastatic breast cancer with lung metastases treated with metastasectomy demonstrated improved median survival (37 vs. 25 months) and five-year survival ( $38 \%$ vs. $18 \%$ ) for patients receiving complete versus incomplete resection $(\mathrm{P}=0.0009)$ (49). Another retrospective series of patients undergoing liver resection for metastatic breast cancer demonstrated improved overall survival (OS) for patients with preoperative disease response or stabilization with systemic therapy compared to patients with preoperative disease progression (80 vs. 30 months, $\mathrm{P}<0.001$ ) (50). Additional studies have reported favorable outcomes of breast cancer patients undergoing resection of brain $(48,51,52)$ and bone (53) metastases as well. While still controversial, in the setting of these data rates of metastasectomy have been increasing across all cancer sites in recent years (6).

\section{Radiation therapy}

In addition to surgery, radiation therapy has been used to treat oligometastatic disease. The most common technique employed is often referred to as stereotactic body radiation therapy (SBRT), stereotactic ablative radiation therapy (SABR), or, perhaps more appropriately, hypofractionated image-guided radiation therapy (HIGRT). This technique utilizes high doses per fraction of highly conformal radiation therapy with steep dose gradients to ablate targeted lesions while sparing normal tissue (54). Numerous studies have demonstrated high treated metastasis control rates with acceptable toxicities for lung (55), liver (56), adrenal (57), and multi-site HIGRT $(58,59)$. Long-term results were recently published for 48 patients with oligometastatic breast cancer treated with HIGRT as a subset of patients enrolled on two prospective trials investigating HIGRT for oligometastatic disease of various primary tumors (URCC 8700 and URCC 9700) (60). In this subset of breast cancer patients, 5- and 10 -year overall survival was $83 \%$ and $75 \%$, respectively, for patients with bone-only oligometastases, while patients with non-bone oligometastases had 5- and 10-year OS of 31\% and $17 \%$, respectively. In light of this emerging evidence, the use of HIGRT to treat oligometastases has increased over time. A recent international survey of over 1,000 radiation oncologists reported over $60 \%$ of practitioners are treating oligometastases with HIGRT, and of those who are not currently doing so, $59 \%$ plan to start (5).

\section{Is oligometastatic breast cancer unique?}

The long natural history of certain metastatic breast cancers, particularly those with hormone receptor positive disease and bone-only metastases, seemingly make them ideal for treatment of all oligometastases with local therapy, as extended progression-free and overall survival may best be demonstrated in the setting of slow disease progression such as in this patient population. One histology agnostic study of 361 patients with extracranial oligometastatic cancers treated with HIGRT from multiple prospective clinical trials sought to identify prognostic pretreatment factors in order to identify which patients may benefit most (61). Median OS was 47.1 months overall, and on univariate analysis patients with breast cancer had significantly longer OS compared to patients with colorectal, other gastrointestinal, NSCLC, sarcoma, and other primary tumor types. On multivariate analysis using Cox proportional hazards models, this OS difference was sustained for breast cancer compared to all but colorectal cancers. The study also used a recursive partitioning analysis (RPA) to identify prognostic classes for overall survival, and this grouped breast, kidney, and prostate cancer into a distinct group from other primary tumor types, with 3 -year OS of $75 \%$ for these patients. RPA was also able to group patients with disease from these three primary sites based on progression-free survival (PFS), demonstrating 3-year PFS of $44 \%$ for this group compared 
to $17 \%$ for other primary sites. Similarly, Wong et al. analyzed prognostic factors in 61 patients treated with oligometastasis-directed HIGRT on a dose-escalation trial (62). They found that patients with breast cancer compared to non-breast cancer had improved OS (median, two-year, and five-year OS of 4.3 years, $100 \%$, and $50 \%$, respectively, vs. 2 years, $52 \%$, and $29 \%$, respectively; $\mathrm{P}=0.026$ ) and PFS (median, two-year, and five-year PFS of 2 years, $57 \%$, and $29 \%$, respectively, versus 4.7 months, $18 \%$, and $10 \%$, respectively; $\mathrm{P}=0.054)$. Interestingly, they also conducted an exploratory analysis in 17 patients that found primary tumor expression levels of three microRNAs (miR-23b, miR-44a, and miR-449b) predicted survival.

Molecular markers such as these may help identify which patients are most likely to benefit from MDT, and in particular hormone receptor status may be particularly important in oligometastatic breast cancer. The aforementioned retrospective study from MD Anderson of 86 patients with metastatic breast cancer who underwent resection of liver metastases found that patients with estrogen receptor (ER) and/or progesterone receptor (PR) positive disease had improved OS compared to patients with ER-/PR- disease (median 76.8 versus 28.3 months; $\mathrm{P}<0.001)$ (50). On multivariate analysis, ER- status was associated with worse overall survival [hazard ratio (HR) 3.3, 95\% confidence interval (CI) 1.4-8.2; $\mathrm{P}=0.009]$. Similarly, a study of 50 patients with extracranial oligometastatic breast cancer treated with metastasis-directed radiation therapy found worse OS for hormone receptor negative patients (HR 7.558, P=0.001), although notably not all of these patients received treatment to all known sites of disease (63). Among patients with oligometastatic breast cancer treated with SBRT on 2 prospective trials at the University of Rochester, ER and/or PR positivity was associated with both improved OS $(\mathrm{P}=0.0009)$ and freedom from widespread metastases $(\mathrm{P}=0.0009)$ in patients with bone-only metastases but not in patients with non-bone metastases (64). Improved outcomes for hormone receptor positive disease are likely due to a combination of more indolent disease biology and administration of hormonal therapy. In the setting of antiHER2 therapies, HER2 status may also be an important prognostic factor in oligometastatic breast cancer treated with MDT, although to our knowledge no studies have specifically evaluated this.

\section{What is the level 1 evidence for MDT of oligometastatic patients?}

For years, evidence for MDT included only retrospective data and small prospective series. However, large randomized trials have recently been published supporting definitive treatment of oligometastatic disease with demonstrated improvements in progression-free and overall survival, as well as decreased development of new metastases (Table 1).

Although difficult to complete, these disease specific trials will need to continue to identify the correct timing, if any, of MDT within a given disease. This is critically important for breast cancer given the long natural history of the disease in some patients and evolving systemic therapies that may alter if or when metastasis-directed therapies are administered.

A multicenter, randomized, phase II trial of patients with metastatic NSCLC with three or fewer metastases and no progression at three or more months after front-line systemic therapy randomized patients to local consolidative therapy (LCT: radiotherapy or surgery) to all active disease sites (primary and metastatic tumors) or to maintenance therapy/observation (MT/O) (10). Radiotherapy with SBRT, HIGRT, and concurrent chemoradiotherapy were allowed, per physician discretion. The trial was closed early after 49 patients enrolled because of a significant PFS benefit in the LCT arm. At long-term median followup of 38.8 months, patients treated with LCT compared to MT/O had significantly greater PFS (median 14.2 vs. 4.4 months, $\mathrm{P}=0.022$ ) and $\mathrm{OS}$ (median 41.2 vs. 17.0 months; $\mathrm{P}=0.017$ ). Interestingly, this trial also demonstrated a trend towards reduction in time to appearance of new metastases in the LCT arm (14.2 vs. 6.0 months, $\mathrm{P}=0.11)$, suggesting a possible systemic benefit to LCT (11), consistent with analyses demonstrating metastases themselves are the source of further metastatic spread (65). Similarly, a phase II trial from UT Southwestern randomized patients with limited metastatic NSCLC (primary plus up to five metastatic sites) to maintenance chemotherapy alone or HIGRT followed by maintenance chemotherapy (12). After 29 patients enrolled with a median follow-up of 9.6 months, the trial was stopped to accrual early after an interim analysis demonstrated significantly improved PFS in the HIGRT arm (9.7 vs. 3.5 months, $\mathrm{P}=0.01$ ). Grade 3 or greater toxicities were similar in both groups, with 4 instances likely treatment related in the HIGRT-plus-maintenance arm and 3 instances likely treatment related in the maintenance chemotherapy alone arm.

Additionally, EORTC 40004 was a randomized phase II trial of 119 patients with fewer than 10 unresectable colorectal liver metastases and no extrahepatic sites of disease. Patients were randomized to receive systemic 


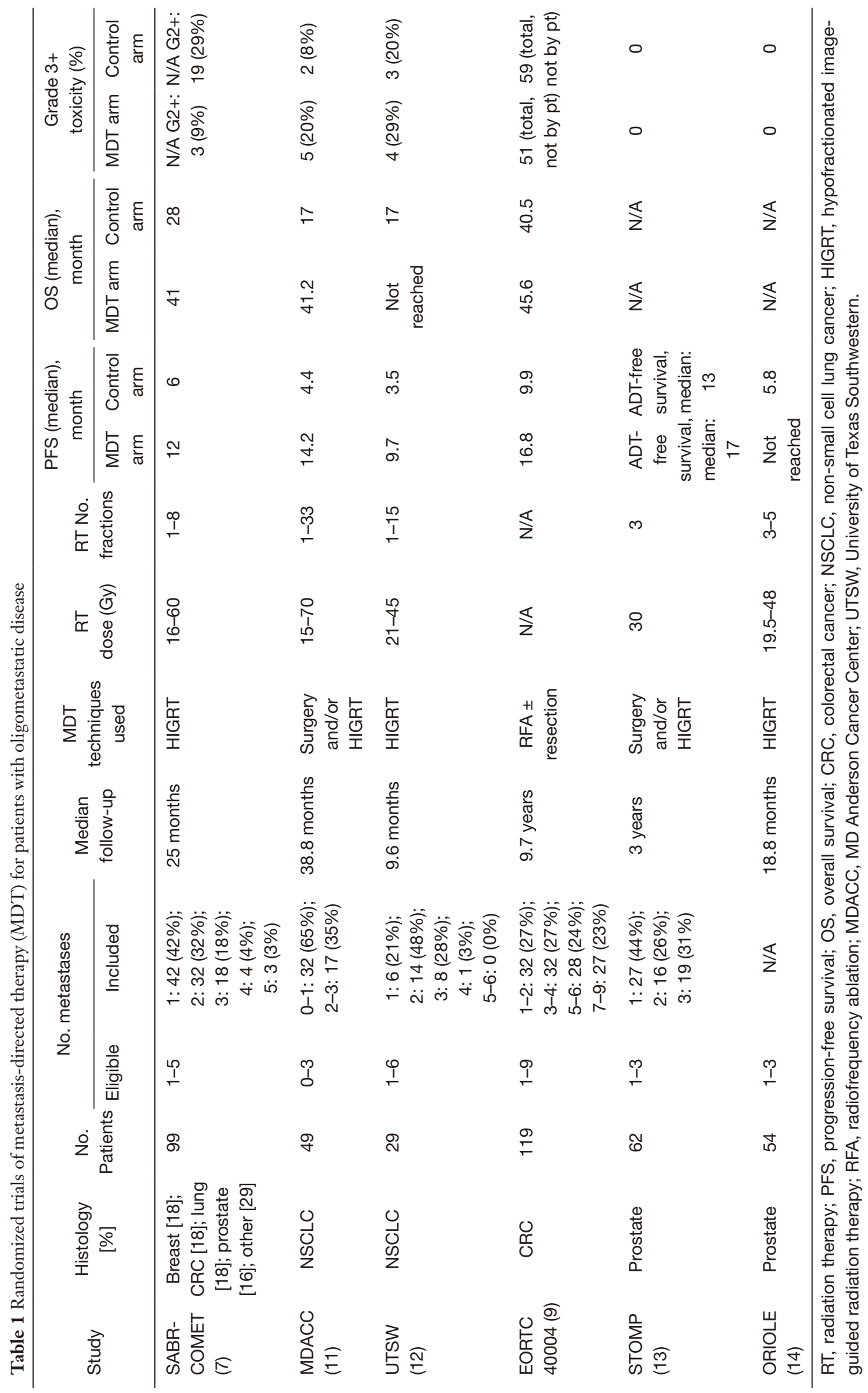


therapy alone or systemic therapy plus aggressive local treatment with radiofrequency ablation (RFA) with or without resection. At median follow-up of 9.7 years, patients in the combined modality arm had significantly increased OS compared to the systemic therapy arm (median $45.6 \mathrm{vs}$. 40.5 months, HR 0.58 , 95\% CI 0.38-0.88; $\mathrm{P}=0.01$ ) (9).

Next, the STOMP study published in 2018 was a multicenter, randomized, phase II trial of patients with biochemically recurrent prostate cancer after definitive treatment with three or fewer detectable extracranial metastases. Patients were randomized to either surveillance or MDT of all known sites of disease. At median followup of three years, the primary end point of androgen deprivation therapy (ADT)-free survival was greater for the treatment group (median 21 vs. 13 months, HR 0.60, $80 \%$ CI, 0.40-0.90, $\mathrm{P}=0.11$ ) (13). Similarly, the recent ORIOLE phase II multicenter trial randomized 54 men with recurrent hormone-sensitive prostate cancer and one to three detectable metastases to HIGRT or observation. The primary outcome of progression at six months was significantly improved for patients receiving HIGRT (19\% vs. $61 \%, \mathrm{P}=0.005)$. No grade three or greater toxicities were observed (14).

Additionally, the SABR-COMET trial was an international, randomized, phase II study of 99 patients with a controlled primary tumor and one to five metastases randomized to either palliative standard of care treatments alone or standard of care plus SABR to all metastatic lesions. While this trial allowed for up to five metastases, only seven patients $(7 \%)$ had more than three, and the median number was two. At median follow-up of over two years, median OS was greater in the SABR group compared to the control (41 vs. 28 months, HR 0.57. 95\% CI, 0.30$1.10, \mathrm{P}=0.09)$. Note, this study used a two-sided alpha of 0.2 , where $\mathrm{P}<0.20$ demonstrates a treatment worth additional investigation. Three (4.5\%) of 66 patients in the SABR arm had treatment-related death (7). Notably, this trial has been criticized for imbalance in primary disease sites between the two groups: the SABR group included more breast and prostate cancers $(20 \%$ and $21 \%$, respectively) compared to the control group (15\% and $6 \%$, respectively), which may bias results as these cancers often have more indolent biology and prolonged natural histories (66).

Lastly, the long-awaited NRG BR001 trial of HIGRT for oligometastases has completed accrual but results have not yet been published. This phase I trial included patients with good performance status with four or fewer metastases amenable to HIGRT from breast, lung, or prostate primaries (67). Thirty-six patients were treated with HIGRT to all known sites of disease and are eligible for analysis. This trial aims to establish the safety of various HIGRT dose schedules in patients with multiple metastases, and accrual has completed and results have been presented in conference proceedings. Given favorable results, treatment of similar patients has since been expanded into the ongoing randomized trials NRG BR002 for oligometastatic breast cancer and LU002 for oligometastatic lung cancer (68).

\section{What is the evidence in breast cancer?}

Over the last two decades, a growing body of nonrandomized data has emerged supporting the use of MDT for oligometastatic breast cancer (Table 2).

As discussed above, retrospective surgical series have demonstrated improved survival in patients with oligometastatic breast cancer treated with metastasectomy for lung and liver metastases, including improved outcomes for patients with hormone receptor positive disease $(49,50)$. Additionally, Trovo et al. conducted a prospective phase II multicenter trial in Italy of 54 patients with oligometastatic breast cancer ( $\leq$ five extracranial metastases) treated with HIGRT (30-45 Gy in 3 fractions) or intensity modulated radiotherapy (IMRT, 60 Gy in 25 fractions) to all known sites of disease. At a median follow-up of 30 months, oneand two-year PFS was $75 \%$ and $53 \%$, respectively, and two-year OS was $95 \%$. No grade three or higher toxicities were reported (69). Additionally, Milano et al. from the University of Rochester analyzed a subset of patients with breast cancer from two prospective trials investigating the use of HIGRT for oligometastatic disease. At initial publication, 40 patients were included, and four-year outcomes for patients treated with curative intent included OS of $59 \%$ and PFS of $38 \%$ (64). At longer term followup, 48 patients were included and they found some patients survived $>10$ years (60). In particular, patients with boneonly metastases had good outcomes, with 5- and 10-year overall survival of $83 \%$ and $75 \%$, respectively, and 5-, and 10 -year freedom from widespread metastases (FFWM) of $67 \%$ and $67 \%$. Patients with non-bone metastases had lower 5 - and 10 -year OS of $31 \%$ and $17 \%$, respectively, and 5 - and 10 -year FFWM of $30 \%$ and $15 \%$, respectively. They also found fewer metastases (one vs. $>$ one) and number of involved organs (one $v s$. > one) to be associated with improved FFWM in patients with non-bone metastases.

On the basis of these data, the NRG BR002 randomized phase II/III trial (NCT02364557) was designed, randomizing patients with oligometastatic breast cancer with controlled locoregional disease and four or fewer 


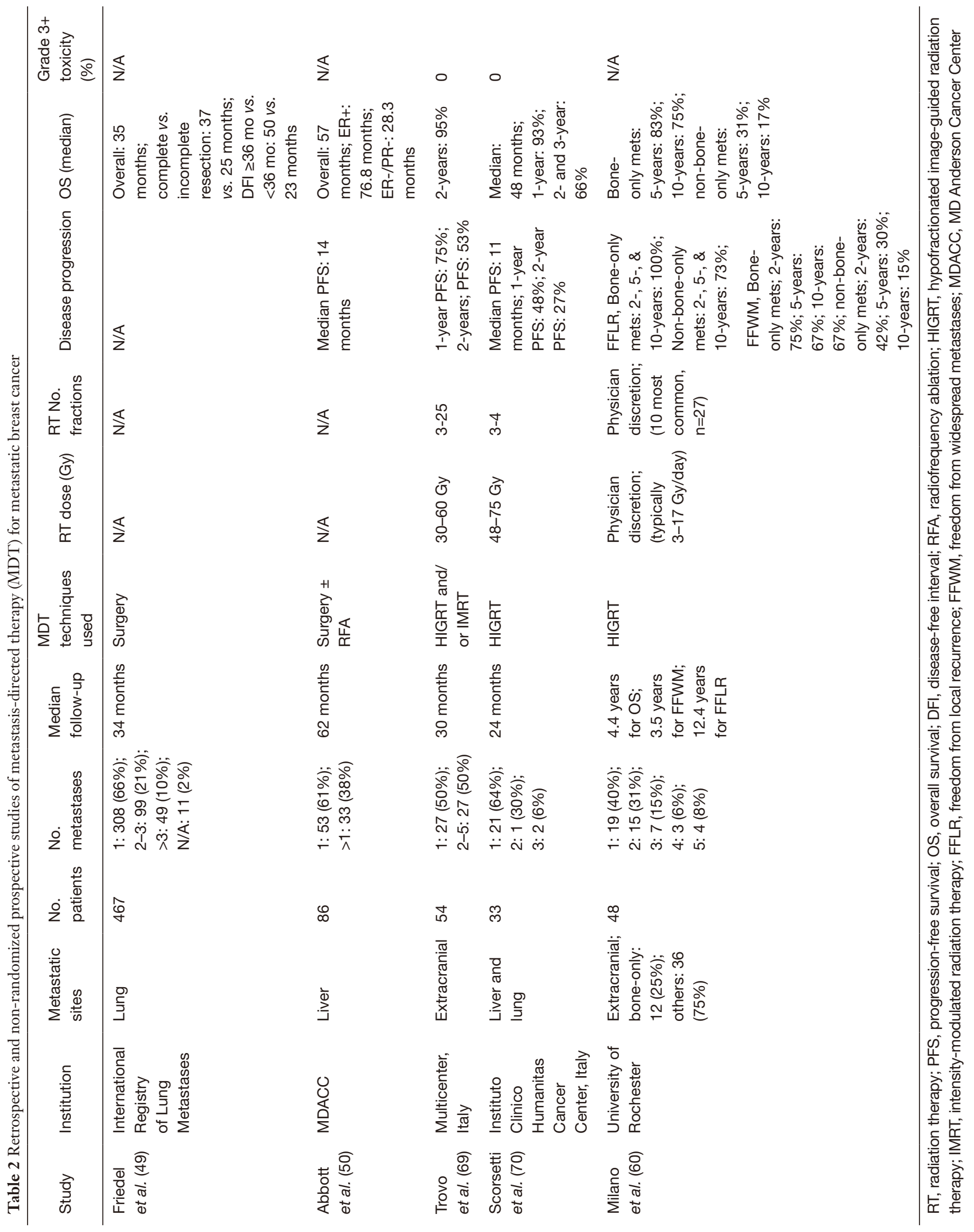


metastases to standard systemic and palliative therapy or ablative therapy (HIGRT or surgery) to all metastases (15). The primary endpoint of the phase II portion is improved PFS, and the primary endpoint of the phase III portion is improved OS. Additionally, circulating tumor cells (CTCs) are collected at baseline, after treatment, and at progression to determine whether CTCs are a predictive marker for improved outcomes. The phase II portion has completed accrual with 128 patients enrolled, and data are maturing. Depending on the results, NRG BR002 may continue to phase III with an accrual goal of an additional 232 patients.

\section{Should patients with de novo oligometastatic disease undergo local therapy in addition to MDT?}

For patients presenting with de novo oligometastatic disease (untreated primary tumor in addition to limited metastases), controversy exists regarding whether patients may benefit from surgery to the primary with or without adjuvant local radiotherapy versus systemic therapy alone. A recent NCDB analysis indicates that $43 \%$ of women diagnosed with stage IV breast cancer between 2003 and 2012 underwent surgical resection within the first year of their diagnosis despite lack of clear benefit (71). A multicenter prospective registry study (TBCRC 013) of patients with metastatic breast cancer analyzed 90 patients classified as responders to first line systemic therapy, 39 of whom subsequently underwent surgery to the primary disease site (72). No difference in 3-year OS was seen between responders who underwent surgery and those who did not $(77 \%$ vs. $76 \%$, $\mathrm{P}=0.85$ ) regardless of ER and HER2 status. While older retrospective series suggested there may be a survival benefit to local therapy in this context (73-77), more recent randomized studies have shown mixed results.

An early study from India (Tata Memorial Hospital) enrolled 716 women with de novo metastatic breast cancer from 2005 to 2013 and randomized those who responded to upfront systemic therapy to receive locoregional treatment with surgery and adjuvant radiation. Median OS was no different in the locoregional treatment group compared to the no-locoregional treatment group (19.2 vs. 20.5 months, $\mathrm{P}=0.79$ ) (78). However, this study has been criticized for suboptimal systemic therapy (e.g., limited taxane use; $92 \%$ of patients with HER2 positive disease did not receive antiHER2 therapy).

A subsequent Turkish study (MF07-01) randomized 274 treatment-naïve patients between 2007 and 2012 with stage
IV breast cancer to receive locoregional treatment (LRT) followed by systemic therapy (ST) versus ST alone (79). At 3 -year follow-up the survival rate was similar between the two groups $(60 \%$ and $51 \%$, respectively, $\mathrm{P}=0.1)$. However, with longer follow-up of 5 years, median survival was significantly improved for patients receiving local therapy, with a median survival of 46 months in the LRT group versus 37 months in the ST group (HR 0.66, 95\% CI, 0.49$0.88, \mathrm{P}=0.005)$. Unplanned subgroup analysis indicated survival advantage to local therapy for patients with ER+/ $\mathrm{PR}+$ disease, HER2/NEU negative disease, patients younger than 55 years of age, and patients with solitary bone-only metastases.

Additional randomized data is expected from the ongoing ECOG E2108 trial (NCT01242800) randomizing women with stage IV breast cancer who have not progressed during initial optimal systemic therapy to receive surgical resection followed by adjuvant radiation in the case of breast conservation and adjuvant radiation following mastectomy at physician discretion. This study is now closed to accrual after enrolling 390 participants. Preliminary data presented at ASCO 2020 demonstrated that at median follow-up of 59 months there was no significant difference between optimal systemic therapy plus locoregional therapy compared to optimal systemic therapy alone for 3 -year OS (68.4\% vs. $67.9 \%$, HR $1.09,90 \%$ CI, $0.80-1.49)$ or PFS $(\mathrm{P}=0.40)$. However, 3-year locoregional recurrence or progression was significantly higher in the systemic therapy alone arm (25.6\% vs. $10.2 \%, \mathrm{P}=0.003)$ (80). Final results are eagerly awaited to help inform locoregional decision making in the setting of de novo metastatic disease.

\section{Do advances in systemic therapy change MDT?}

In addition to more aggressive use of surgery and radiation directed at specific metastases, recent improvements in systemic therapies are altering the disease course of breast cancer and have potential implications on how and when to incorporate MDT. For example, the recent CLEOPATRA study changed standard of care for patients with metastatic HER2 positive breast cancer after demonstrating improved OS and PFS for patients receiving pertuzumab, trastuzumab, and docetaxel compared to placebo, trastuzumab, and docetaxel $(81,82)$. In hormone receptor positive, HER2 negative metastatic breast cancer, cyclindependent kinase 4/6 inhibitors including palbociclib, ribociclib, and abemaciclib, are now being used in addition to hormonal therapy after multiple randomized phase III 
trials demonstrated their use improves PFS and/or OS (16). Other targeted agents such as PI3K and PARP inhibitors are also being incorporated into the treatment of metastatic breast cancer to improve outcomes (17-20).

While systemic therapy is improving progression-free survival in patients with metastatic breast cancer, longterm complete remission with systemic therapy alone is rare (83-85). However, as these newer systemic therapies prolong the disease course, more providers may reasonably consider aggressive MDT with curative intent in patients with oligometastatic breast cancer. Progression in these patients often occurs at sites of known metastases rather than new metastatic locations $(59,60)$, and therefore ablation of all known sites of cancer may maximize therapeutic benefit. Alternatively, some may say that we should hold off on MDT until all systemic options are depleted.

\section{What about immunotherapy?}

Immune checkpoint inhibitors have recently been incorporated into the treatment of certain metastatic triple-negative breast cancers after results of the Impassion 130 trial showed improved PFS and OS in patients with high PD-L1 tumor expression treated with nab-paclitaxel plus atezolizumab compared to patients receiving nabpaclitaxel alone (22). Additionally, in the non-metastatic setting, promising early results from the KEYNOTE-173 phase III trial of patients with stage II-III triple negative breast cancer treated with paclitaxel and carboplatin with or without pembrolizumab showed improved pathologic complete response in the pembrolizumab-chemotherapy group $(64.8 \%$ vs. $51.2 \%, \mathrm{P}<0.001)(21)$. As immunotherapy becomes more commonplace in treatment algorithms for breast cancer patients, studies investigating its combination with HIGRT have demonstrated the combination to be safe and well tolerated. For example, a study of 79 patients treated with this combination had only 6 dose-limiting toxicities and no radiation dose reductions (86).

Beyond having an acceptable safety profile, radiation therapy may have an immune-mediated effect that works synergistically with immunotherapy. The so-called "abscopal effect," in which radiotherapy to one site produces a response at a distant, non-irradiated site, was first described by Mole in 1953 and has since been reported multiple times in the literature, including in combination with immune checkpoint inhibitors (87-89). Ablative-doses of radiotherapy have been shown to have effects mediated by CD8+ T cells, as well as to increase type I interferon responses $(90,91)$. Based on these preclinical data, there has been growing interest in combining HIGRT with immunotherapy. For example, a phase I study investigated the use of HIGRT in either one, two, or three doses (20 Gy per fraction), followed by high-dose interleukin-2 (IL-2) for patients with metastatic melanoma or renal cell carcinoma. The combination was safe and demonstrated better-thanexpected response rates in melanoma patients $(71.4 \% \mathrm{PR}$ or CR), hypothesized to be due to an immune-mediated response (92). However, to our knowledge the combination of HIGRT with immunotherapy has not been studied yet in breast cancer specifically, although in other diseases there are promising preliminary reports $(93,94)$.

\section{Are there non-curative benefits of HIGRT?}

In addition to potentially improving OS and PFS, metastasis-directed HIGRT may have benefits such as symptom palliation and delaying systemic therapy. For patients who may typically receive palliative radiotherapy for painful bone metastases, HIGRT can similarly provide good pain control and potentially long-term local control (95). HIGRT for bone metastases has been shown to be well tolerated with limited grade three or greater toxicities (96). Additionally, a phase II/III randomized trial (RTOG 0631) investigated pain relief for painful bone metastases treated with HIGRT to the involved spine segments(s) versus conventional external beam radiotherapy to the involved spine plus one additional segment above and below (97). Initial phase III results demonstrated HIGRT was safe and well tolerated but showed no difference in pain relief at three months post-treatment between the two groups (98).

Another potential benefit to HIGRT for oligometastases and oligoprogressive lesions is delaying initiation of systemic therapy or transitioning to next-line therapy. The STOMP trial of patients with oligometastatic prostate cancer randomized to surveillance or MDT to all known sites of disease showed significantly longer ADTfree survival for the treatment group (13). Ranck et al. demonstrated that in patients with oligometastatic renal cell carcinoma, $64.2 \%$ of patients treated with metastasisdirected SBRT did not require systemic therapy at one year (99). HIGRT may be particularly useful in elderly patients who may not be candidates for or decline other treatment modalities, and HIGRT has been shown to have excellent rates of local control in these patients $(86.8 \%$ at one year, $76.3 \%$ at two years) (70). Given these findings, HIGRT to oligometastases should be considered in certain 
situations even in non-curative settings.

\section{So which patients should be treated?}

A number of factors must be considered when deciding which patients with oligometastatic breast cancer should be treated with metastasis-directed therapies, and this is an active area of investigation. Importantly, we await the results of the NRG BR002 phase III trial to help answer this question. In the meantime, one factor that should be considered is the number of metastatic sites and organs. Most trials, such as SABR-COMET have included patients with five or fewer metastases, although only $7 \%$ of patients on this trial had more than three metastases. Milano et al. found that both number of metastases and number of involved organs involved ( $1 v s .>1)$ were associated with improved OS and FFWM in patients with oligometastatic breast cancer with non-bone metastases treated with ablative MDT (60). Additionally, they showed improved OS and PFS in patients with bone-only metastases compared to non-bone metastases, suggesting these patients may warrant more aggressive therapy. Additionally, certain patients with hormone receptor positive disease may have a prolonged disease course in the setting of endocrine therapy, and ER+/ $\mathrm{PR}+$ disease has been shown to be associated with improved outcomes after metastasis-directed $(50,60)$. Other potentially prognostic factors such as tumor microRNA expression and CTCs are under active investigation $(15,62)$.

\section{Conclusions}

MDT is an effective treatment to control limited metastatic disease and potentially improve survival and progressionfree survival in patients with oligometastatic breast cancer. High level, randomized data has emerged to support this approach for other primary disease sites, and research is ongoing for oligometastatic breast cancer specifically. MDT may be especially useful in breast cancer given its long natural history, particularly in the setting of improved systemic therapies including targeted agents and immunotherapy that prolong the disease course. Some patients with oligometastatic breast cancer treated with ablative therapy to all sites of disease have survival $>10$ years and may even be cured of their disease (60). Finally, the role of MDT in this patient population will be further clarified by the results of the ongoing NRG BR002 phase II/III randomized trial, and we eagerly await these results.

\section{Acknowledgments}

Funding: None.

\section{Footnote}

Provenance and Peer Review: This article was commissioned by the Guest Editors (Simon Lo, Michael Milano, Tithi Biswas, Charles Simone) for the series "OligometastasisFallacy or Real Deal?" published in Annals of Palliative Medicine. The article has undergone external peer review.

Reporting Checklist: The authors have completed the NARRATIVE REVIEW reporting checklist. Available at http://dx.doi.org/10.21037/apm-20-1128

Conflicts of Interest: All authors have completed the ICMJE uniform disclosure form (available at http://dx.doi. org/10.21037/apm-20-1128). The series "OligometastasisFallacy or Real Deal?” was commissioned by the editorial office without any funding or sponsorship. The authors have no other conflicts of interest to declare.

Ethical Statement: The authors are accountable for all aspects of the work in ensuring that questions related to the accuracy or integrity of any part of the work are appropriately investigated and resolved.

Open Access Statement: This is an Open Access article distributed in accordance with the Creative Commons Attribution-NonCommercial-NoDerivs 4.0 International License (CC BY-NC-ND 4.0), which permits the noncommercial replication and distribution of the article with the strict proviso that no changes or edits are made and the original work is properly cited (including links to both the formal publication through the relevant DOI and the license). See: https://creativecommons.org/licenses/by-nc-nd/4.0/.

\section{References}

1. Fisher B. Laboratory and clinical research in breast cancer-a personal adventure: the David A. Karnofsky memorial lecture. Cancer Res 1980;40:3863-74.

2. Stephens SJ, Moravan MJ, Salama JK. Managing Patients With Oligometastatic Non-Small-Cell Lung Cancer. J Oncol Pract 2018;14:23-31.

3. Salama JK, Milano MT. Radical irradiation of extracranial 
oligometastases. J Clin Oncol 2014;32:2902-12.

4. Hong JC, Salama JK. The expanding role of stereotactic body radiation therapy in oligometastatic solid tumors: What do we know and where are we going? Cancer Treat Rev 2017;52:22-32.

5. Lewis SL, Porceddu S, Nakamura N, et al. Definitive Stereotactic Body Radiotherapy (SBRT) for Extracranial Oligometastases: An International Survey of $>1000$ Radiation Oncologists. Am J Clin Oncol 2017;40:418-22.

6. Bartlett EK, Simmons KD, Wachtel H, et al. The rise in metastasectomy across cancer types over the past decade. Cancer 2015;121:747-57.

7. Palma DA, Olson R, Harrow S, et al. Stereotactic ablative radiotherapy versus standard of care palliative treatment in patients with oligometastatic cancers (SABRCOMET): a randomised, phase 2, open-label trial. Lancet 2019;393:2051-8.

8. Palma DA, Olson R, Harrow S, et al. Stereotactic Ablative Radiotherapy for the Comprehensive Treatment of Oligometastatic Cancers: Long-Term Results of the SABR-COMET Phase II Randomized Trial. J Clin Oncol 2020;38:2830-8.

9. Ruers T, Van Coevorden F, Punt CJ, et al. Local Treatment of Unresectable Colorectal Liver Metastases: Results of a Randomized Phase II Trial. J Natl Cancer Inst 2017;109:djx015.

10. Gomez DR, Blumenschein GR Jr, Lee JJ, et al. Local consolidative therapy versus maintenance therapy or observation for patients with oligometastatic non-smallcell lung cancer without progression after first-line systemic therapy: a multicentre, randomised, controlled, phase 2 study. Lancet Oncol 2016;17:1672-82.

11. Gomez DR, Tang C, Zhang J, et al. Local Consolidative Therapy Vs. Maintenance Therapy or Observation for Patients With Oligometastatic Non-Small-Cell Lung Cancer: Long-Term Results of a Multi-Institutional, Phase II, Randomized Study. J Clin Oncol 2019;37:1558-65.

12. Iyengar P, Wardak Z, Gerber DE, et al. Consolidative Radiotherapy for Limited Metastatic Non-Small-Cell Lung Cancer: A Phase 2 Randomized Clinical Trial. JAMA Oncol 2018;4:e173501.

13. Ost P, Reynders D, Decaestecker K, et al. Surveillance or Metastasis-Directed Therapy for Oligometastatic Prostate Cancer Recurrence: A Prospective, Randomized, Multicenter Phase II Trial. J Clin Oncol 2018;36:446-53.

14. Phillips R, Shi WY, Deek M, et al. Outcomes of Observation vs Stereotactic Ablative Radiation for Oligometastatic Prostate Cancer: The ORIOLE Phase 2
Randomized Clinical Trial. JAMA Oncol 2020;6:650-9.

15. Chmura SJ, Winter KA, Al-Hallaq HA, et al. NRGBR002: A phase IIR/III trial of standard of care therapy with or without stereotactic body radiotherapy (SBRT) and/or surgical ablation for newly oligometastatic breast cancer (nct02364557). j clin oncol 2019;37:TPS1117.

16. Gao JJ, Cheng J, Bloomquist E, et al. CDK4/6 inhibitor treatment for patients with hormone receptor-positive, HER2-negative, advanced or metastatic breast cancer: a US Food and Drug Administration pooled analysis. Lancet Oncol 2020;21:250-60.

17. Cristofanilli M, Turner NC, Bondarenko I, et al. Fulvestrant plus palbociclib versus fulvestrant plus placebo for treatment of hormone-receptor-positive, HER2negative metastatic breast cancer that progressed on previous endocrine therapy (PALOMA-3): final analysis of the multicentre, double-blind, phase 3 randomised controlled trial. Lancet Oncol 2016;17:425-39.

18. Mehta RS, Barlow WE, Albain KS, et al. Combination anastrozole and fulvestrant in metastatic breast cancer. $\mathrm{N}$ Engl J Med 2012;367:435-44.

19. Litton JK, Rugo HS, Ettl J, et al. Talazoparib in Patients with Advanced Breast Cancer and a Germline BRCA Mutation. N Engl J Med 2018;379:753-63.

20. Robson ME, Tung N, Conte P, et al. OlympiAD final overall survival and tolerability results: Olaparib versus chemotherapy treatment of physician's choice in patients with a germline BRCA mutation and HER2-negative metastatic breast cancer. Ann Oncol 2019;30:558-66.

21. Schmid P, Cortes J, Pusztai L, et al. Pembrolizumab for Early Triple-Negative Breast Cancer. N Engl J Med 2020;382:810-21.

22. Schmid P, Rugo HS, Adams S, et al. Atezolizumab plus nab-paclitaxel as first-line treatment for unresectable, locally advanced or metastatic triple-negative breast cancer (IMpassion130): updated efficacy results from a randomised, double-blind, placebo-controlled, phase 3 trial. Lancet Oncol 2020;21:44-59.

23. Hellman S. Karnofsky Memorial Lecture. Natural history of small breast cancers. J Clin Oncol 1994;12:2229-34.

24. Hellman S, Weichselbaum RR. Oligometastases. J Clin Oncol 1995;13:8-10.

25. Cheruvu P, Metcalfe SK, Metcalfe J, et al. Comparison of outcomes in patients with stage III versus limited stage IV non-small cell lung cancer. Radiat Oncol 2011;6:80.

26. Lecouvet FE, Oprea-Lager DE, Liu Y, et al. Use of modern imaging methods to facilitate trials of metastasisdirected therapy for oligometastatic disease in prostate 
cancer: a consensus recommendation from the EORTC Imaging Group. Lancet Oncol 2018;19:e534-45.

27. deSouza NM, Liu Y, Chiti A, et al. Strategies and technical challenges for imaging oligometastatic disease: Recommendations from the European Organisation for Research and Treatment of Cancer imaging group. Eur J Cancer 2018;91:153-63.

28. Guckenberger M, Lievens Y, Bouma AB, et al. Characterisation and classification of oligometastatic disease: a European Society for Radiotherapy and Oncology and European Organisation for Research and Treatment of Cancer consensus recommendation. Lancet Oncol 2020;21:e18-28.

29. Niibe Y, Hayakawa K. Oligometastases and oligorecurrence: the new era of cancer therapy. Jpn J Clin Oncol 2010;40:107-11.

30. Weickhardt AJ, Scheier B, Burke JM, et al. Local ablative therapy of oligoprogressive disease prolongs disease control by tyrosine kinase inhibitors in oncogene-addicted non-small-cell lung cancer. J Thorac Oncol 2012;7:1807-14.

31. Gan GN, Weickhardt AJ, Scheier B, et al. Stereotactic radiation therapy can safely and durably control sites of extra-central nervous system oligoprogressive disease in anaplastic lymphoma kinase-positive lung cancer patients receiving crizotinib. Int J Radiat Oncol Biol Phys 2014;88:892-8.

32. Sledge GW, Neuberg D, Bernardo P, et al. Phase III trial of doxorubicin, paclitaxel, and the combination of doxorubicin and paclitaxel as front-line chemotherapy for metastatic breast cancer: an intergroup trial (E1193). J Clin Oncol 2003;21:588-92.

33. Albain KS, Nag SM, Calderillo-Ruiz G, et al. Gemcitabine plus Paclitaxel versus Paclitaxel monotherapy in patients with metastatic breast cancer and prior anthracycline treatment. J Clin Oncol 2008;26:3950-7.

34. Gianni L, Romieu GH, Lichinitser M, et al. AVEREL: a randomized phase III Trial evaluating bevacizumab in combination with docetaxel and trastuzumab as first-line therapy for HER2-positive locally recurrent/metastatic breast cancer. J Clin Oncol 2013;31:1719-25.

35. Hurvitz SA, Dirix L, Kocsis J, et al. Phase II randomized study of trastuzumab emtansine versus trastuzumab plus docetaxel in patients with human epidermal growth factor receptor 2-positive metastatic breast cancer. J Clin Oncol 2013;31:1157-63.

36. Tawfik H, Rostom Y, Elghazaly H. All-oral combination of vinorelbine and capecitabine as first-line treatment in
HER2/Neu-negative metastatic breast cancer. Cancer Chemother Pharmacol 2013;71:913-9.

37. Bergh J, Bondarenko IM, Lichinitser MR, et al. Firstline treatment of advanced breast cancer with sunitinib in combination with docetaxel versus docetaxel alone: results of a prospective, randomized phase III study. J Clin Oncol 2012;30:921-9.

38. Torok JA, Gu L, Tandberg DJ, et al. Patterns of Distant Metastases After Surgical Management of Non-Small-cell Lung Cancer. Clin Lung Cancer 2017;18:e57-70.

39. Singh D, Yi WS, Brasacchio RA, et al. Is there a favorable subset of patients with prostate cancer who develop oligometastases? Int J Radiat Oncol Biol Phys 2004;58:3-10.

40. Howard JH, Thompson JF, Mozzillo N, et al. Metastasectomy for distant metastatic melanoma: analysis of data from the first Multicenter Selective Lymphadenectomy Trial (MSLT-I). Ann Surg Oncol 2012;19:2547-55.

41. Douillard JY, Siena S, Cassidy J, et al. Randomized, phase III trial of panitumumab with infusional fluorouracil, leucovorin, and oxaliplatin (FOLFOX4) versus FOLFOX4 alone as first-line treatment in patients with previously untreated metastatic colorectal cancer: the PRIME study. J Clin Oncol 2010;28:4697-705.

42. Hortobagyi GN, Smith TL, Legha SS, et al. Multivariate analysis of prognostic factors in metastatic breast cancer. J Clin Oncol 1983;1:776-86.

43. Dorn PL, Meriwether A, LeMieux M, et al. Patterns of distant failure and progression in breast cancer: Implications for the treatment of oligometastatic disease. Int J Radiat Oncol Biol Phys 2011. doi: 10.1016/ j.ijrobp.2011.06.1901.

44. Mehta N, Mauer AM, Hellman S, et al. Analysis of further disease progression in metastatic non-small cell lung cancer: implications for locoregional treatment. Int J Oncol 2004;25:1677-83.

45. Pastorino U, Buyse M, Friedel G, et al. Long-term results of lung metastasectomy: prognostic analyses based on 5206 cases. J Thorac Cardiovasc Surg 1997;113:37-49.

46. Fong Y, Cohen AM, Fortner JG, et al. Liver resection for colorectal metastases. J Clin Oncol 1997;15:938-46.

47. Tanvetyanon T, Robinson LA, Schell MJ, et al. Outcomes of adrenalectomy for isolated synchronous versus metachronous adrenal metastases in non-small-cell lung cancer: a systematic review and pooled analysis. J Clin Oncol 2008;26:1142-7.

48. Wro ski M, Arbit E, Burt M, et al. Survival after surgical 
treatment of brain metastases from lung cancer: a followup study of 231 patients treated between 1976 and 1991. J Neurosurg 1995;83:605-16.

49. Friedel G, Pastorino U, Ginsberg RJ, et al. Results of lung metastasectomy from breast cancer: prognostic criteria on the basis of 467 cases of the International Registry of Lung Metastases. Eur J Cardiothorac Surg 2002;22:335-44.

50. Abbott DE, Brouquet A, Mittendorf EA, et al. Resection of liver metastases from breast cancer: estrogen receptor status and response to chemotherapy before metastasectomy define outcome. Surgery 2012;151:710-6.

51. Pieper DR, Hess KR, Sawaya RE. Role of surgery in the treatment of brain metastases in patients with breast cancer. Ann Surg Oncol 1997;4:481-90.

52. Wro ski M, Arbit E, McCormick B. Surgical treatment of 70 patients with brain metastases from breast carcinoma. Cancer 1997;80:1746-54.

53. Dürr HR, Muller PE, Lenz T, et al. Surgical treatment of bone metastases in patients with breast cancer. Clin Orthop Relat Res 2002:191-6.

54. Benedict SH, Yenice KM, Followill D, et al. Stereotactic body radiation therapy: the report of AAPM Task Group 101. Med Phys 2012;39:563.

55. Rusthoven KE, Kavanagh BD, Burri SH, et al. Multiinstitutional phase I/II trial of stereotactic body radiation therapy for lung metastases. J Clin Oncol 2009;27:1579-84.

56. Rusthoven KE, Kavanagh BD, Cardenes H, et al. Multiinstitutional phase I/II trial of stereotactic body radiation therapy for liver metastases. J Clin Oncol 2009;27:1572-8.

57. Torok J, Wegner RE, Burton SA, et al. Stereotactic body radiation therapy for adrenal metastases: a retrospective review of a noninvasive therapeutic strategy. Future Oncol 2011;7:145-51.

58. Milano MT, Katz AW, Zhang H, et al. Oligometastases treated with stereotactic body radiotherapy: long-term follow-up of prospective study. Int J Radiat Oncol Biol Phys 2012;83:878-86.

59. Salama JK, Hasselle MD, Chmura SJ, et al. Stereotactic body radiotherapy for multisite extracranial oligometastases: final report of a dose escalation trial in patients with 1 to 5 sites of metastatic disease. Cancer 2012;118:2962-70.

60. Milano MT, Katz AW, Zhang H, et al. Oligometastatic breast cancer treated with hypofractionated stereotactic radiotherapy: Some patients survive longer than a decade. Radiother Oncol 2019;131:45-51.

61. Hong JC, Ayala-Peacock DN, Lee J, et al. Classification for long-term survival in oligometastatic patients treated with ablative radiotherapy: A multi-institutional pooled analysis. PLoS One 2018;13:e0195149.

62. Wong AC, Watson SP, Pitroda SP, et al. Clinical and molecular markers of long-term survival after oligometastasis-directed stereotactic body radiotherapy (SBRT). Cancer 2016;122:2242-50.

63. Yoo GS, Yu JI, Park W, et al. Prognostic factors in breast cancer with extracranial oligometastases and the appropriate role of radiation therapy. Radiat Oncol J 2015;33:301-9.

64. Milano MT, Zhang H, Metcalfe SK, et al. Oligometastatic breast cancer treated with curativeintent stereotactic body radiation therapy. Breast Cancer Res Treat 2009;115:601-8.

65. Gundem G, Van Loo P, Kremeyer B, et al. The evolutionary history of lethal metastatic prostate cancer. Nature 2015;520:353-7.

66. Macbeth F, Treasure T. Points to consider regarding the SABR-COMET trial. Lancet 2020;395:e19.

67. Al-Hallaq HA, Chmura S, Salama JK, et al. Rationale of technical requirements for NRG-BR001: The first NCIsponsored trial of SBRT for the treatment of multiple metastases. Pract Radiat Oncol 2016;6:e291-8.

68. Chmura SJ, Winter K, Salama JK, et al. Phase I Trial of Stereotactic Body Radiation Therapy (SBRT) to Multiple Metastatic Sites: A NRG Oncology Study. Int J Radiat Oncol Biol Phys 2018;102:S68-9.

69. Trovo M, Furlan C, Polesel J, et al. Radical radiation therapy for oligometastatic breast cancer: Results of a prospective phase II trial. Radiother Oncol 2018;126:177-80.

70. Scorsetti M, Clerici E, Navarria P, et al. The role of stereotactic body radiation therapy (SBRT) in the treatment of oligometastatic disease in the elderly. Br J Radiol 2015;88:20150111.

71. Lane WO, Thomas SM, Blitzblau RC, et al. Surgical Resection of the Primary Tumor in Women With De Novo Stage IV Breast Cancer: Contemporary Practice Patterns and Survival Analysis. Ann Surg 2019;269:537-44.

72. King TA, Lyman J, Gonen M, et al. A prospective analysis of surgery and survival in stage IV breast cancer (TBCRC 013). J Clin Oncol 2017;34:1006.

73. Gnerlich J, Jeffe DB, Deshpande AD, et al. Surgical removal of the primary tumor increases overall survival in patients with metastatic breast cancer: analysis of the 19882003 SEER data. Ann Surg Oncol 2007;14:2187-94.

74. Rapiti E, Verkooijen HM, Vlastos G, et al. Complete 
excision of primary breast tumor improves survival of patients with metastatic breast cancer at diagnosis. J Clin Oncol 2006;24:2743-9.

75. Hazard HW, Gorla SR, Scholtens D, et al. Surgical resection of the primary tumor, chest wall control, and survival in women with metastatic breast cancer. Cancer 2008;113:2011-9.

76. Petrelli F, Barni S. Surgery of primary tumors in stage IV breast cancer: an updated meta-analysis of published studies with meta-regression. Med Oncol 2012;29:3282-90.

77. Khan SA, Stewart AK, Morrow M. Does aggressive local therapy improve survival in metastatic breast cancer? Surgery 2002;132:620-6; discussion 626-7.

78. Badwe R, Hawaldar R, Nair N, et al. Locoregional treatment versus no treatment of the primary tumour in metastatic breast cancer: an open-label randomised controlled trial. Lancet Oncol 2015;16:1380-8.

79. Soran A, Ozmen V, Ozbas S, et al. Randomized Trial Comparing Resection of Primary Tumor with No Surgery in Stage IV Breast Cancer at Presentation: Protocol MF07-01. Ann Surg Oncol 2018;25:3141-9.

80. Khan SA, Zhao F, Solin LJ, et al. A randomized phase III trial of systemic therapy plus early local therapy versus systemic therapy alone in women with de novo stage IV breast cancer: A trial of the ECOG-ACRIN Research Group (E2108). J Clin Oncol 2020;38:suppLBA2.

81. Swain SM, Baselga J, Kim SB, et al. Pertuzumab, trastuzumab, and docetaxel in HER2-positive metastatic breast cancer. N Engl J Med 2015;372:724-34.

82. Swain SM, Miles D, Kim SB, et al. Pertuzumab, trastuzumab, and docetaxel for HER2-positive metastatic breast cancer (CLEOPATRA): end-of-study results from a double-blind, randomised, placebo-controlled, phase 3 study. Lancet Oncol 2020;21:519-30.

83. Greenberg PA, Hortobagyi GN, Smith TL, et al. Longterm follow-up of patients with complete remission following combination chemotherapy for metastatic breast cancer. J Clin Oncol 1996;14:2197-205.

84. Yamamoto N, Katsumata N, Watanabe T, et al. Clinical characteristics of patients with metastatic breast cancer with complete remission following systemic treatment. Jpn J Clin Oncol 1998;28:368-73.

85. Tomiak E, Piccart M, Mignolet F, et al. Characterisation of complete responders to combination chemotherapy for advanced breast cancer: a retrospective EORTC Breast Group study. Eur J Cancer 1996;32A:1876-87.

86. Luke JJ, Lemons JM, Karrison TG, et al. Safety and Clinical Activity of Pembrolizumab and Multisite
Stereotactic Body Radiotherapy in Patients With Advanced Solid Tumors. J Clin Oncol 2018;36:1611-8.

87. Dewan MZ, Galloway AE, Kawashima N, et al. Fractionated but not single-dose radiotherapy induces an immune-mediated abscopal effect when combined with anti-CTLA-4 antibody. Clin Cancer Res 2009;15:5379-88.

88. Golden EB, Demaria S, Schiff PB, et al. An abscopal response to radiation and ipilimumab in a patient with metastatic non-small cell lung cancer. Cancer Immunol Res 2013;1:365-72.

89. Demaria $\mathrm{S}, \mathrm{Ng} \mathrm{B}$, Devitt $\mathrm{M}$, et al. Ionizing radiation inhibition of distant untreated tumors (abscopal effect) is immune mediated. Int J Radiat Oncol Biol Phys 2004;58:862-70.

90. Lee Y, Auh SL, Wang Y, et al. Therapeutic effects of ablative radiation on local tumor require $\mathrm{CD} 8+\mathrm{T}$ cells: changing strategies for cancer treatment. Blood 2009;114:589-95.

91. Burnette BC, Liang H, Lee Y, et al. The efficacy of radiotherapy relies upon induction of type i interferondependent innate and adaptive immunity. Cancer Res 2011;71:2488-96.

92. Seung SK, Curti BD, Crittenden M, et al. Phase 1 study of stereotactic body radiotherapy and interleukin-2-tumor and immunological responses. Sci Transl Med 2012;4:137ra74.

93. Theelen W, Peulen HMU, Lalezari F, et al. Effect of Pembrolizumab After Stereotactic Body Radiotherapy vs Pembrolizumab Alone on Tumor Response in Patients With Advanced Non-Small Cell Lung Cancer: Results of the PEMBRO-RT Phase 2 Randomized Clinical Trial. JAMA Oncol 2019;5:1276-82.

94. Bauml JM, Mick R, Ciunci C, et al. Pembrolizumab After Completion of Locally Ablative Therapy for Oligometastatic Non-Small Cell Lung Cancer: A Phase 2 Trial. JAMA Oncol 2019;5:1283-90.

95. Jhaveri PM, Teh BS, Paulino AC, et al. A doseresponse relationship for time to bone pain resolution after stereotactic body radiotherapy (SBRT) for renal cell carcinoma (RCC) bony metastases. Acta Oncol 2012;51:584-8.

96. Owen D, Laack NN, Mayo CS, et al. Outcomes and toxicities of stereotactic body radiation therapy for non-spine bone oligometastases. Pract Radiat Oncol 2014;4:e143-9.

97. Ryu S, Pugh SL, Gerszten PC, et al. RTOG 0631 Phase II/III Study of Image-Guided Stereotactic Radiosurgery for Localized (1-3) Spine Metastases: Phase II Results. Int 
J Radiat Oncol Biol Phys 2011;81:S131-2.

98. Ryu S DS, Timmerman RD, Movsas B, et al. Radiosurgery Compared To External Beam Radiotherapy for Localized Spine Metastasis: Phase III Results of NRG Oncology/
RTOG 0631. Int J Radiat Oncol Biol Phys 2019;105:S2-3. 99. Ranck MC, Golden DW, Corbin KS, et al. Stereotactic body radiotherapy for the treatment of oligometastatic renal cell carcinoma. Am J Clin Oncol 2013;36:589-95.

Cite this article as: Kent CL, McDuff SGR, Salama JK. Oligometastatic breast cancer: where are we now and where are we headed?-a narrative review. Ann Palliat Med 2021;10(5):59545968. doi: 10.21037/apm-20-1128 